\title{
MEDICIÓN DE NIVEL EN LOS MOLDES DE COLADA CONTINUA*
}

\section{Resumen}

Raúl Daniel López ${ }^{1}$ Jaime Usart Daniel Cerutti

Un objetivo de seguridad y calidad en los moldes de la colada continua de Siderar fue la de tener un menisco estable y controlado. A lo largo de los distintos periodos de inversiones y mejoras, el control de nivel de Siderar logro como objetivo contener su variación de nivel de acero en el molde en un rango de variación menor a $+/-2$ $\mathrm{mm}$ y con una velocidad de cambio inferior a $1,5 \mathrm{~m} /$ segundos. Esta etapa trajo una relativa mejora en los aspectos mencionados (calidad y continuidad operativa) pero el hecho de tener como sistema de medición de nivel un sistema radiactivo (fuente Co60 y contador de centelleo) mantenía serias limitaciones en ambos rubros dado que este sistema no diferencia la interface escoria /polvo colador con el menisco de acero. Este trabajo tratará de mostrar y contar las tareas de desarrollo y aplicación de esta nueva tecnología, hoy vigente en la nueva Colada Continua № 2 (CCD2) y en etapa de puesta en marcha de la CCD1.

Palabras-clave: Medición electromagnética; Medición radiactiva; Sensor suspendido; Sensor a bordo del molde.

\section{STEEL LEVEL MEASUREMENT IN CONTINUOUS CASTER MOULD \\ Abstract}

One safety and quality aim in the continuous caster mold at Siderar was to have a stable and controlled meniscus. Throughout the different investment and improvement periods, the level control at Siderar achieved its aim to hold the steel level in the mould in a variation range lower than $+/-2 \mathrm{~mm}$ with a change speed lower than $1.5 \mathrm{~m} / \mathrm{sec}$. This stage brought about a relative improvement in the already mentioned aspects (quality and operational continuity), but the fact of having a radioactive system to measure (Co60 source and sparkling counter) kept both fields limited, as this system does not distinguish the slag/casting powder interface from the steel meniscus. This paper intends to show and describe the development and application tasks of this new technology, which is now present at the new Continuous Caster №2 and on the way of being implemented on the Continuous Caster №1.

Keywords: Electromagnetic measurement; radioactive measurement; Hanging sensor; Sensor on board of the mould.

1 Ingeniero Electrónico, Coordinador de Mejoras, Asistencia Técnica, Ternium-Siderar, San Nicolás, Provincia de Buenos Aires, República Argentina.

2 Ingeniero Mecánico, Tecnólogo Proyectos de Acería, Ingeniería y Medio ambiente, TerniumSiderar, San Nicolás, Provincia de Buenos Aires, República Argentina.

3 Ingeniero Electrónico, Ingeniero de Proyecto, Automación y Control, San Nicolás, Provincia de Buenos Aires, República Argentina. 


\section{INTRODUCCIÓN}

El control de nivel de acero en el molde que esta operando en la colada continua $\mathrm{N}^{\circ} 1$ de Siderar esta basado en un diseño en conjunto con SMS-DEMAG y Siderar [1] y la corrección del modelo de aplicación especifica con asistencias técnicas de ARVA (Arthur Waterlaus) [2].

Este sistema respeta como idea básica la de tener un menisco estable, de hecho los elementos que constituyen el control de esta variable son de alta velocidad y aplicación de control tipo robótica para garantizar un menisco controlado.

La figura a continuación (Figura 1), muestra la cantidad de variables que pueden generar inestabilidad y turbulencia en el nivel de acero del molde (Menisco) de una colada continua

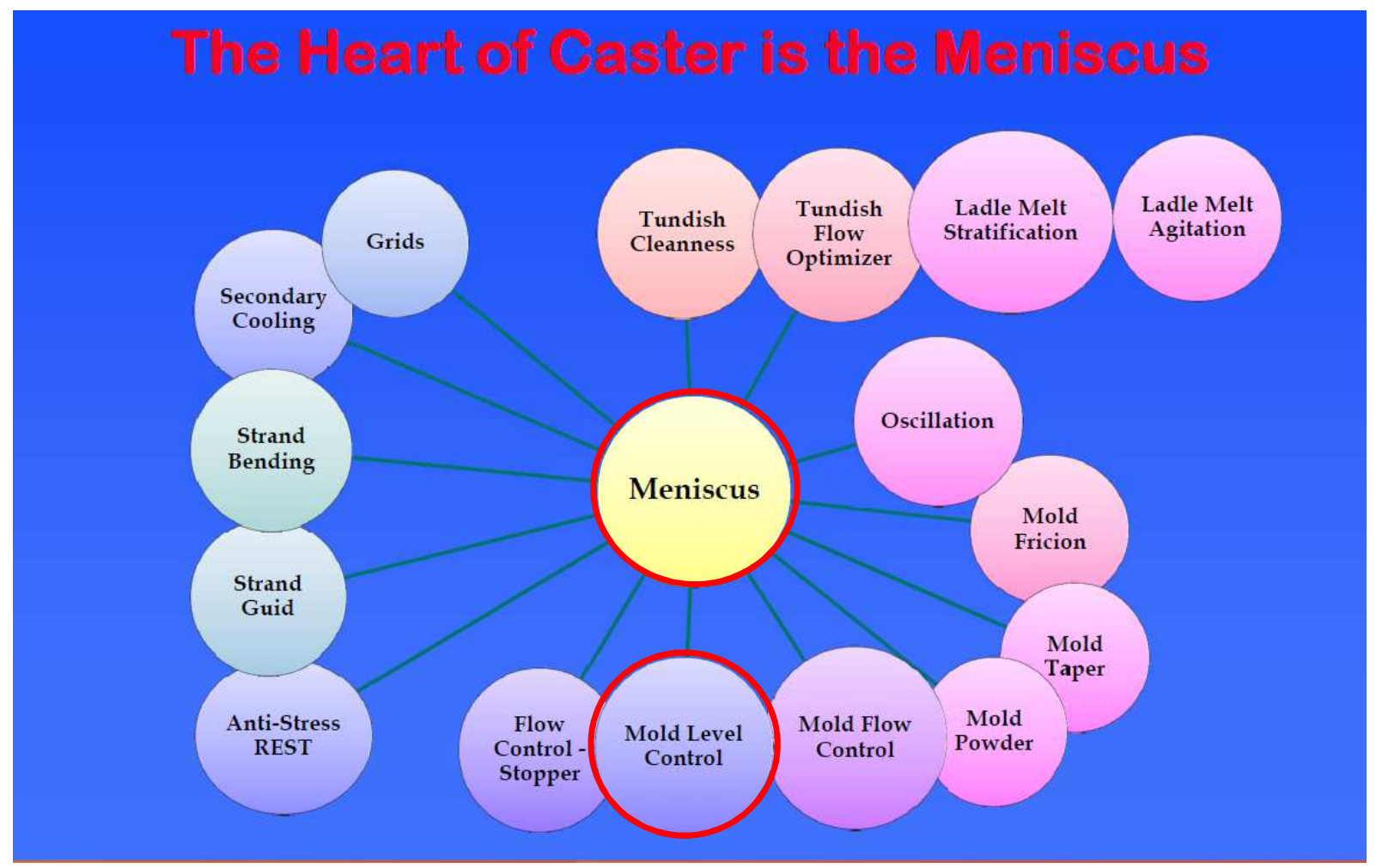

Figura 1. El Menisco es el corazón de la colada

En este trabajo se va a comentar como se instrumentó el "Control de Nivel" de acero en el molde para poder correlacionarse con el resto de las variables mostradas en la figura con la idea básica la de tener un menisco estable y controlado ante las fluctuaciones del resto de las variables que de hecho son dinámicas durante el proceso de colado.

En la (Figura 2) se muestra el estado real del menisco durante el proceso de inicio de solidificación con los grandes riesgos de posible atrapes de cordones de polvo colador aún no fundido como así también los denominados "anillos de escoria", fuentes principales de "break-out" en caso de no controlados.

El sistema esta basado en dos lazos PID (Figura 3) que se comportan como Master Slave, el primero es el encargado de evaluar la señal de nivel de acero, procesarla y controlarla según fuente de Set-Point recibido. El lazo esclavo recibe esta señal y como tal tiene la función de realizar un control inteligente del tapón para permitir el caudal de acero necesario según la demanda del desvío (error) de nivel en cada instante. 


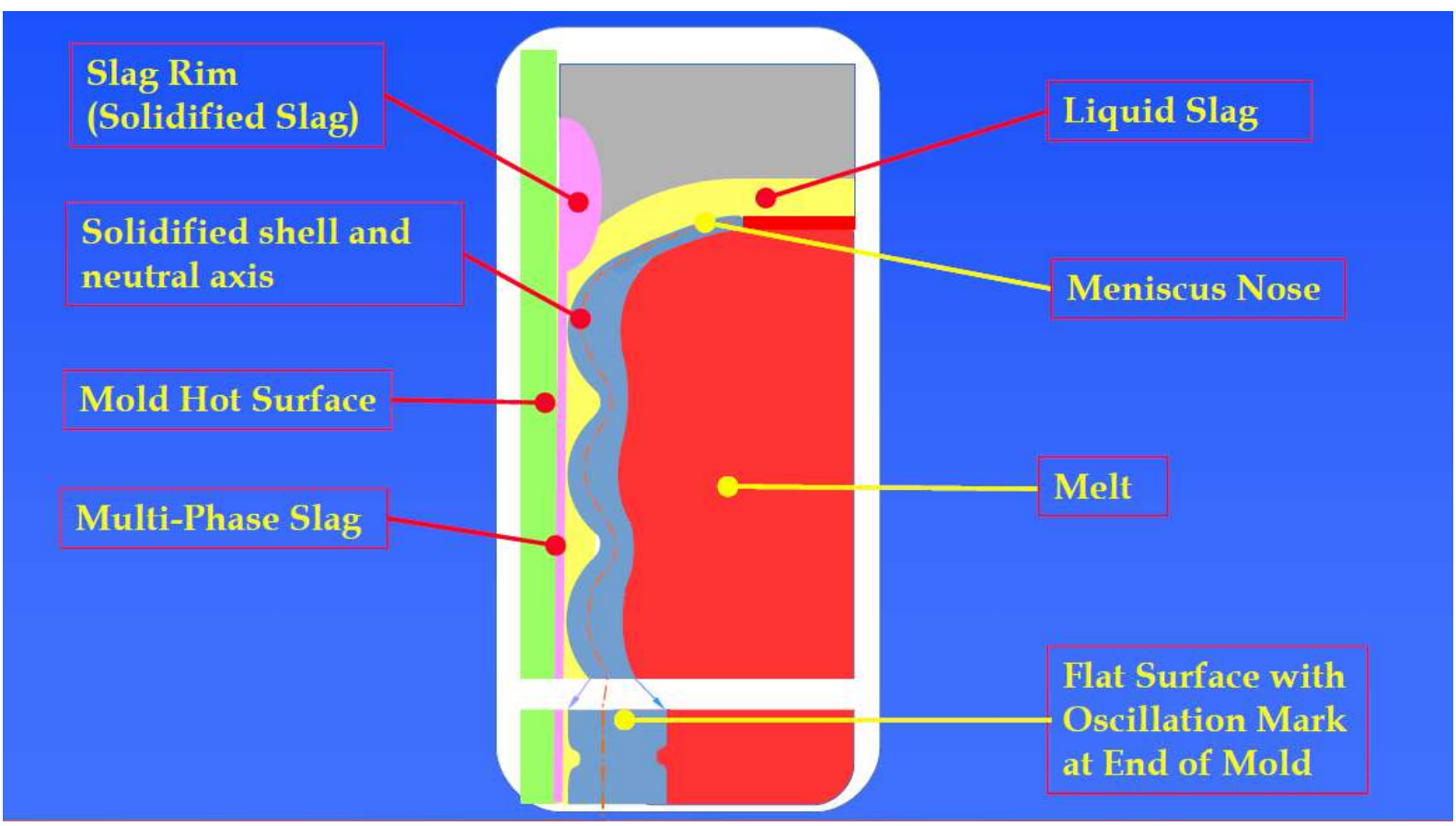

Figura 2. Esquema estado real del menisco

Las consignas de este control de nivel es la de permitir variaciones de amplitud menores a $+/-2 \mathrm{~mm}$ y con una velocidad de cambio inferior a $1,5 \mathrm{~mm} /$ segundos.

Se aclara que nuestro sistema esta instalado en una maquina de colado de planchones de dos líneas, la misma tiene montador moldes curvos de espesor $200 \mathrm{~mm}$, longitud $900 \mathrm{~mm}$ y con sistema de ajuste automático del ancho RAM, durante el colado permite ajuste de 830 a $1630 \mathrm{~mm}$, como así también un sistema de detector de adherencia MSD montado en sus placas de cobre.

\section{Master}

\section{Slave}

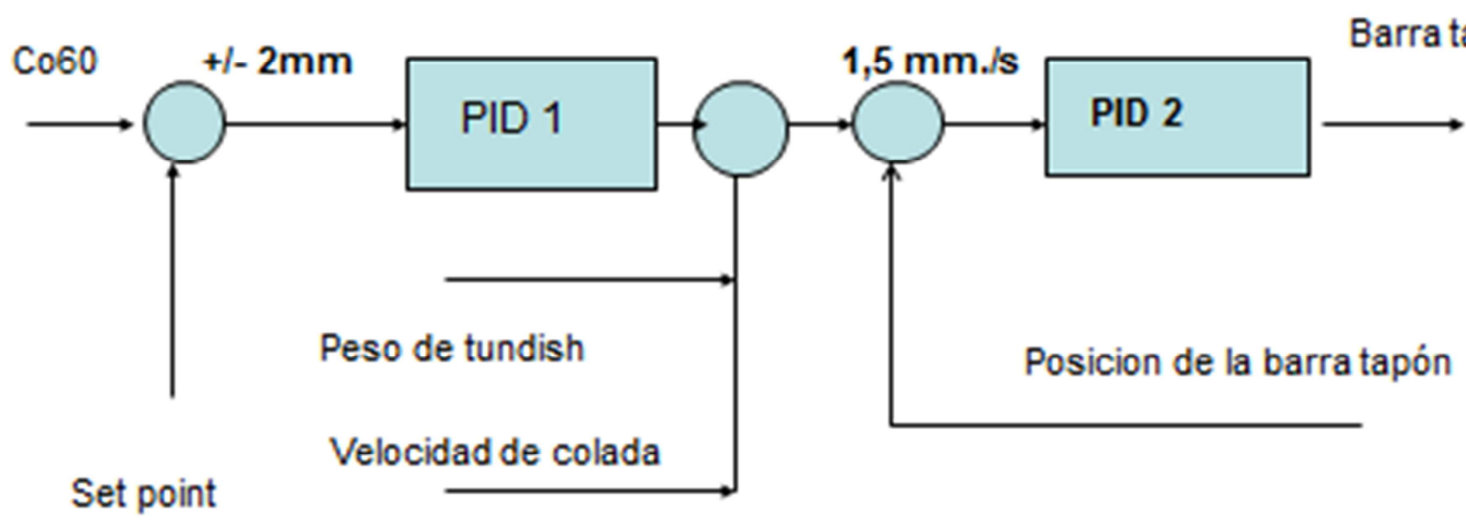

Figura 3. Control de nivel con comando de trayectoria brazo porta tapón.

El elemento de acción final es la barra tapón, la misma es accionado por un brazo Porta-Stopper con accionamiento hidráulico y ambos sistema están montados en nuestros repartidores (Tundish de $36 \mathrm{t}$ )

A continuación se muestra un esquema inicial de nuestro control de nivel, en el mismo esta agregado las funciones de validación para correcciones antes cambios de velocidad de colado y variación de presión ferrostática por peso en repartidor). 


\section{MEDICION DE NIVEL DE ACERO A PARTIR SENSOR ELECTROMAGNETICO}

Una segunda etapa para complementar la aplicación fue la llevada a cabo en instalar en nuestros moldes un sistema con brazos manuales de sensores electromagnéticos, en dicha oportunidad la idea base fue el diseño NKK pero se comercializó con la firma MPC un producto que a priori nos permitía el manejo de esta variable

El objetivo de esta etapa fue la de controlar el verdadero nivel de menisco, la medición con Co60 tiene el limitante que la lectura de la variable de proceso es un promedio de acero mas escoria y polvo colado, con lo que el caudal de acero que se envía a la variable controlada es siempre errático.

\subsection{EXPERIENCIAS REALIZADAS CON SENSORES ELECTROMAGNÉTICOS SUSPENDIDOS}

1) En este caso (Figura 4) se muestra un gráfica controlando nivel de acero en el molde con sensor suspendido.

La grafica color amarillo (Figura 5) representa el nivel de menisco leído y controlado por el sensor electromagnético, la grafica color azul es la lectura del Co60, se observa que por encima del nivel de acero están los umbrales de escoria y polvo colado que son aportados manualmente y consumidos /fundidos durante el proceso de solidificación.

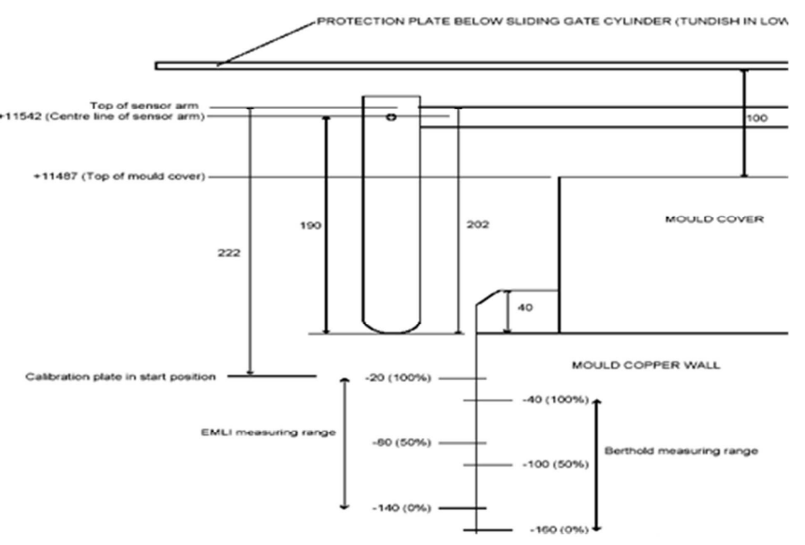

Figura 4. Control con sensor suspendido

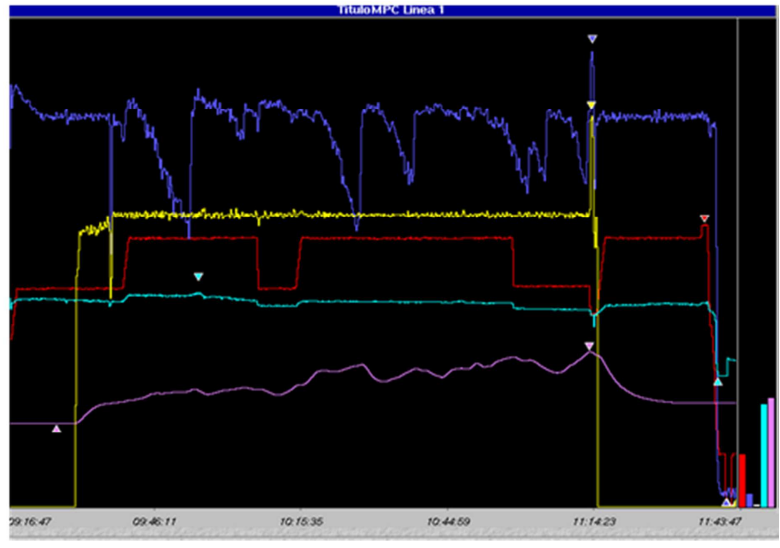

Figura 5. Trending de variables

2) En el caso de las (Figuras 6 y 7), se muestra la actividad del menisco cuando el sistema de control de acero en el molde es a partir del sistema Co60. Se destaca que la lectura promedio permanece constante en el tiempo (gráfica azul de Co60) pero el menisco leído/interpretado por el sensor electromagnético (grafica color amarillo) muestra las variaciones que sufre ante cada aporte manual de polvo colador por parte del operador.

Por ultimo la (Figura 8) muestra una situación donde el control de nivel siempre en AUTO está controlando con Co60, luego con maniobra manual previa pasa a AUTO con sensor electromagnético y luego se retoma la activad original.

Este sistema fue implementado en el año 2006, pero las interferencias en el posicionado del sensor, la actividad ascenso y descenso dinámico de nuestros repartidores, generó muchas interferencias en el posicionado como el retiro del sensor en operación. También el gran nivel de roturas y lo frágil de la cobertura del sensor primario nos llevó a discontinuar con esta aplicación. 


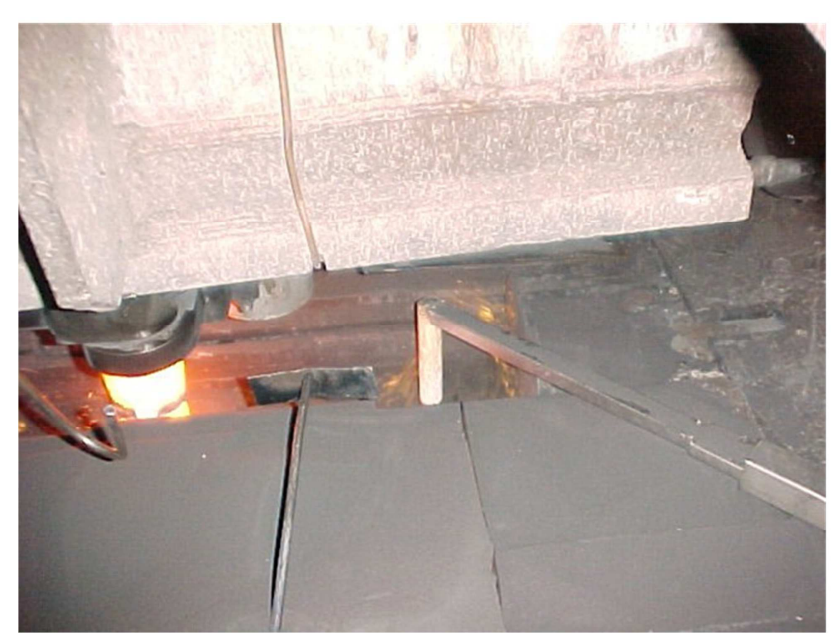

Figura 6. Sensor suspendido

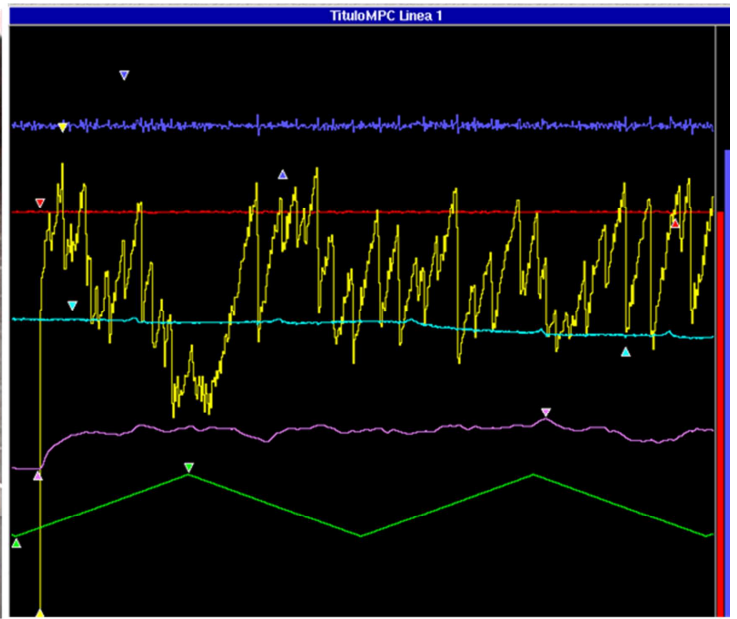

Figura 7. Trending de variables

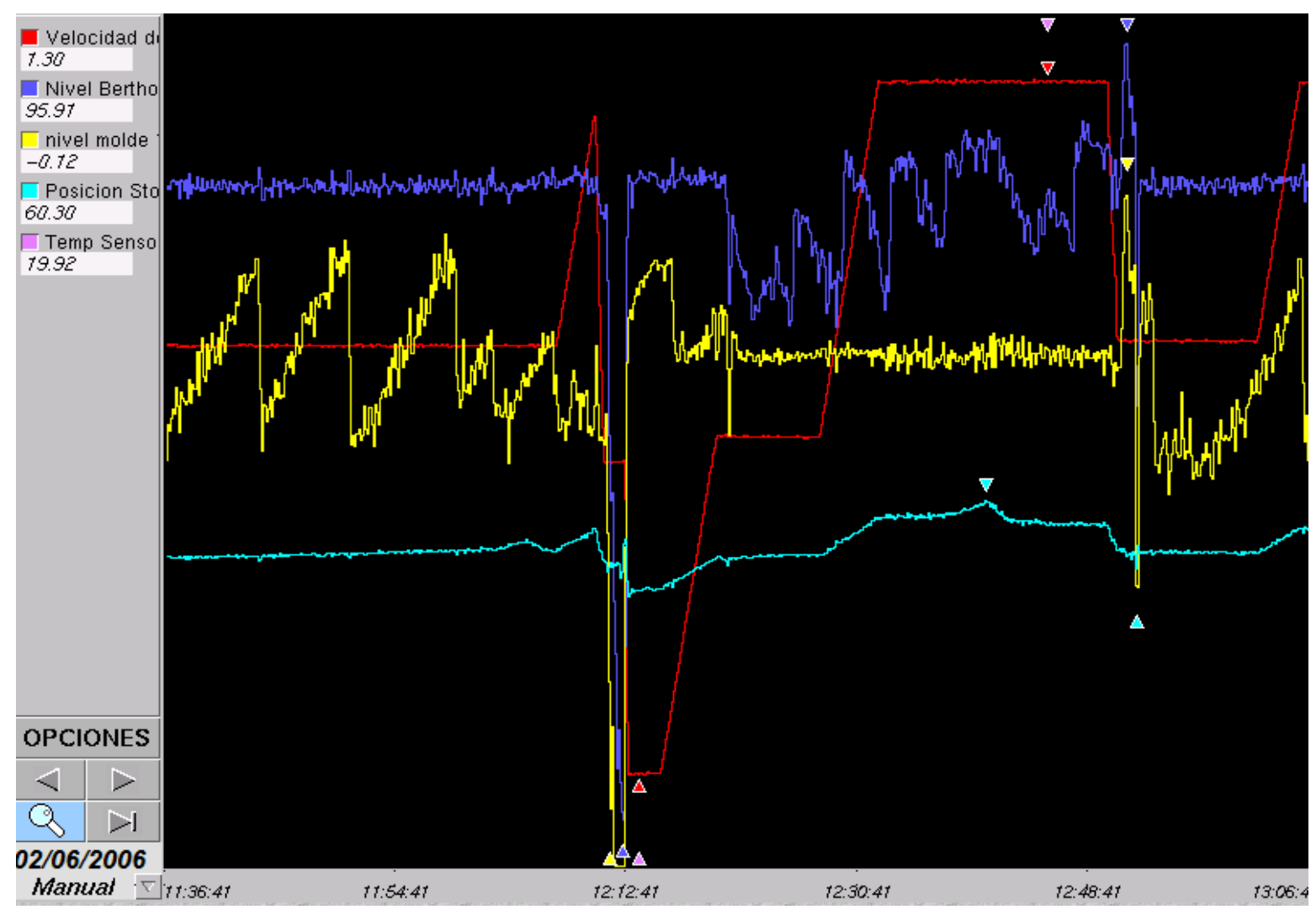

Figura 8. Trending de variables: "Secuencia Manual-Auto"

\section{.3 MEDICION DE NIVEL DE ACERO EN EL MOLDE CON SENSOR A BORDO}

En el año 2008, junto con la especificación de la nueva colada continua de SIDERAR a instalar (CCD2) con molde recto, se empieza a evaluar la posibilidad de utilizar un diseño fijo sobre la caja de agua de nuestras placas para los moldes existentes en nuestra CCD1.

Esta aplicación tenia varias fuentes comerciales SERT, VUHZ, incluso MPC también la suministraba, pero lo concreto, para esta etapa se necesita adaptar cajas de agua 
de nuevo diseño, sistema de refrigeración del sensor a bordo del molde y electrónica complementaria para tratar esta variable.

\section{CAJAS DE AGUA NUEVO DISEÑO PARA REFRIGERACION ESPESOR 200 CON MONTAJE SENSOR VUHZ}

La primera etapa para esta nueva aplicación fue la de rediseñar las cajas de aguas para los actuales moldes de la CCD1 y poder adaptar en ellos este nueva tecnología (sensor electromagnético incorporado). Un detalle de este nuevo diseño se observa en la (Figura 9).

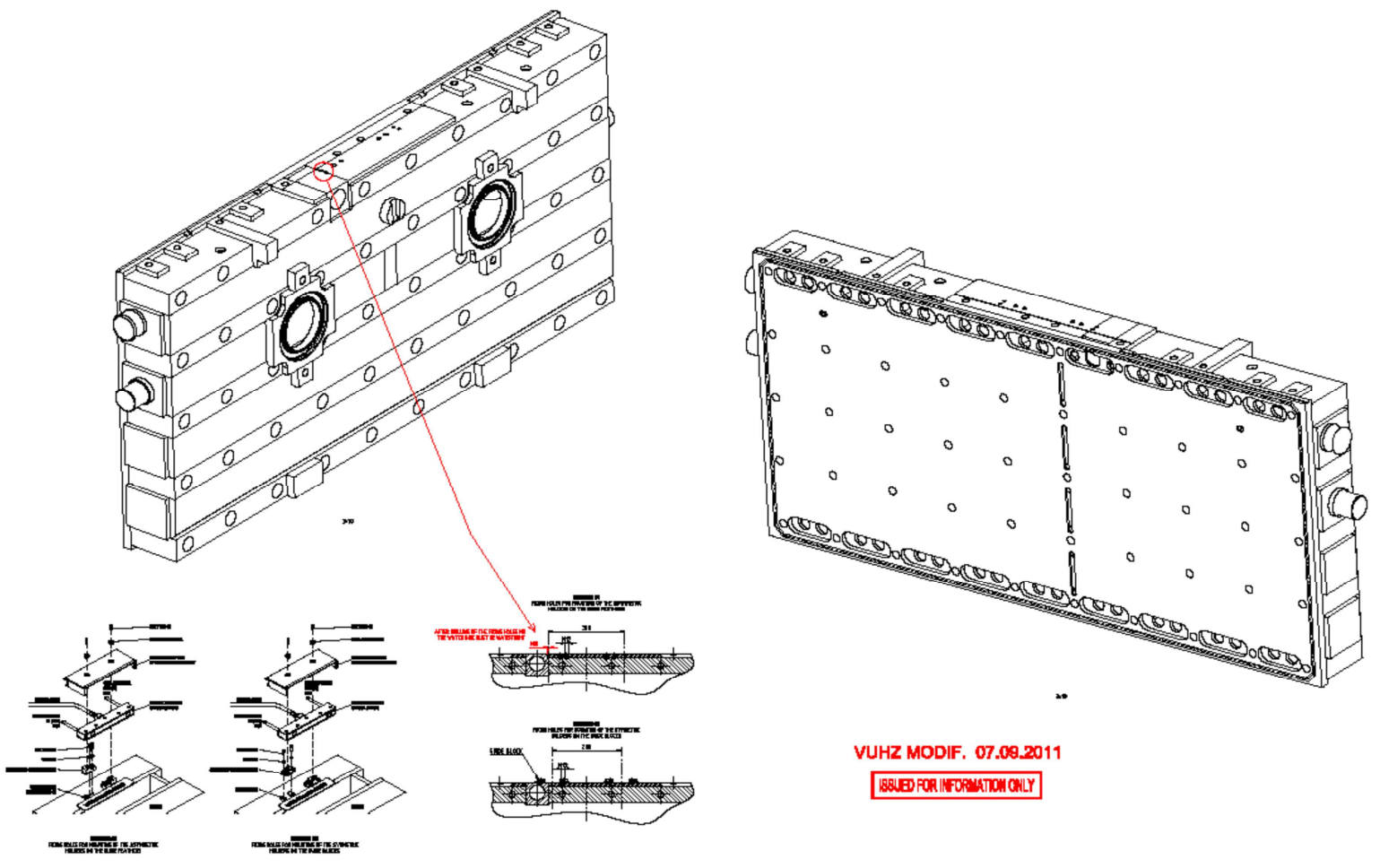

Figura 9. Nuevas Cajas de Agua

Esta etapa fue todo un desafío, pues el nuevo diseño de cajas de agua también permitió reducir una gran cantidad de eventos que persistían en la fijación de estas cajas con las placas de cobre, reducir las perdidas de agua como así también minimizar la deformación de éstas posterior a sus campañas de colado.

La compra se implementó a través de una inversión, se compraron los seis juegos de cajas de agua tanto ancha fija y móvil como las cajas de agua para las placas laterales. El diseño consultado con SMS, la construcción y puesta en marcha demando casi cuatro años.

\section{IMPLEMENTACION TAREAS DE PUESTA EN MARCHA SISTEMA VUHZ}

1) Compra de sistema de medición (tres unidades electrónicas) una para cada línea y otra para stand taller zonal.

2) Montaje sistema de refrigeración hidráulica de los sensores. Se realizo durante las tareas de "Paradas Programadas" de la CCD1 instalando la alimentación de agua de circuito cerrado, intercalando paneles de instrumentación asociado. (Figura10).

3) Se acondicionó circuito de alimentación eléctrico/hidráulico de los moldes para alojar a los sensores. (Figura 12). 
4) Se realizo ingeniería y montaje eléctrico para interconectar nuevo sistema de medición al Sistema de Nivel 1.

La Figura 10 también muestra el sistema de piping que se llevó desde el ingreso en cero mts del sistema de agua de cerrado de maquina (Nivel de Piso), pasando por plataforma osciladores de moldes hasta acometida superior de plataforma de colado. La Figura 11 muestra el montaje del sensor y su correspondiente distribución circuito de refrigeración y alimentación eléctrica.

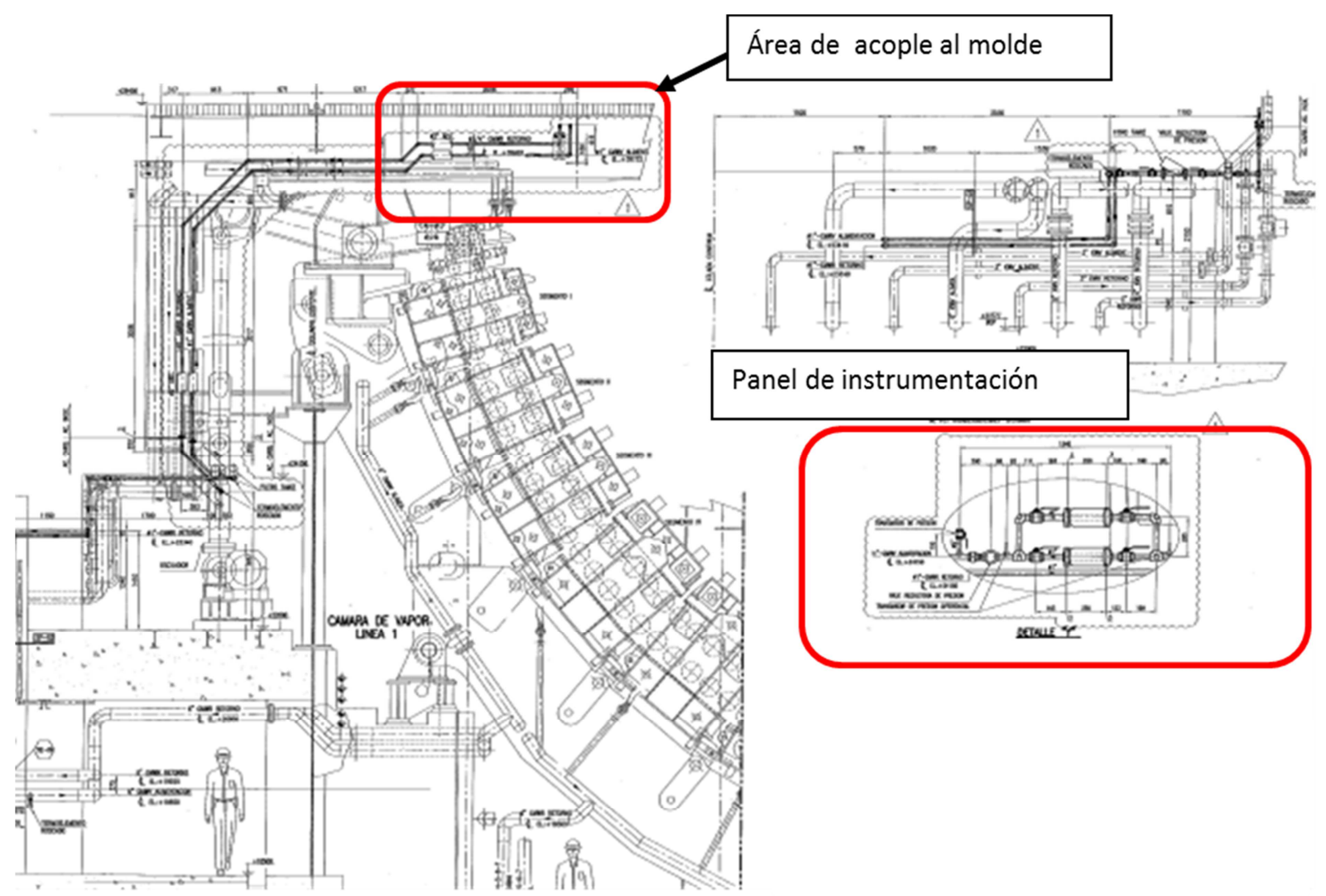

Figura 10. Sistema de refrigeración en la Línea

Por ultimo la Figura 12 muestra el nuevo hardware del sistema. En el mismo se observa que la electrónica de nivel 1 que maneja el lazo Master comparte dos electrónicas de medición de nivel, una unidad llamada de evaluación del VUHZ y una segunda unidad de evaluación de Co60 .Estas unidades son selectables según el modo de control utilizado por el operador para manejar el lazo en automático.

El segundo lazo no sufre variaciones relevantes, sigue con su electrónica dedicada a controlar los Stopper a través de los paneles hidráulicos de alta precisión.

A continuación se muestras graficas de mediciones de nivel con sensores electromagnéticos en la CCD1, este sistema esta operando en forma solo visualización desde el día 16 de Julio 2014, en las próximas "Paradas" se pasaría a los ajuste de Nivel 1 para que el sistema pueda operar en modo automático.

La grafica (Figura 13) muestra un inicio de secuencia en línea №2 de CCD1 con molde $\mathrm{N}^{\circ} 8$ con sensor VUHZ a bordo.

La gráfica siguiente (Figura 14) es un zoom de la anterior, en ésta se observa que, mientras el nivel mostrado por Co60 permaneces constante, las lecturas del sensor electromagnético sufre y muestra las verdaderas variaciones del menisco en este caso por el aporte manual de polvo colador. 

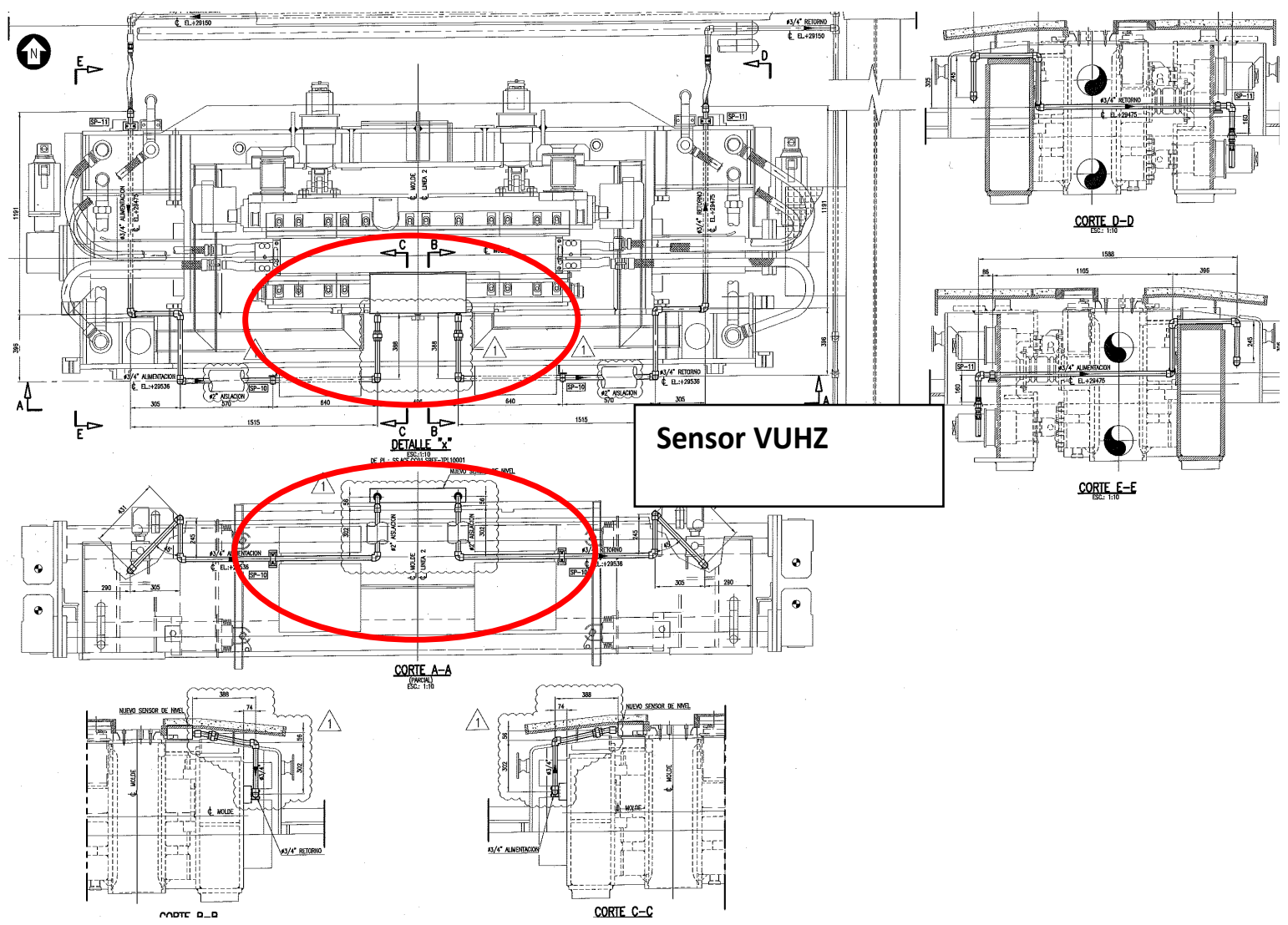

Figura 11. Sistema de refrigeración en el Molde

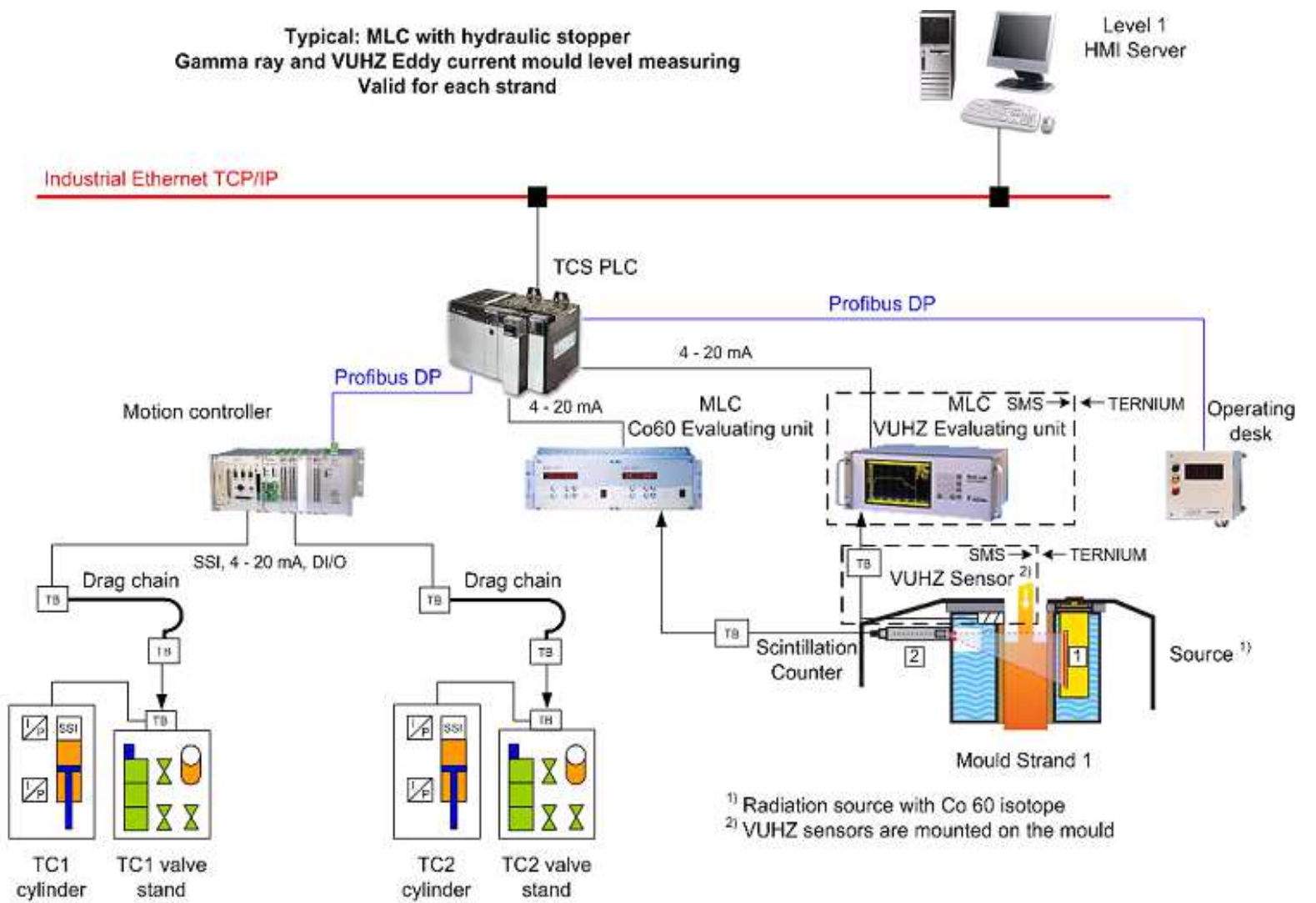

Figura 12. Estructura de Control 


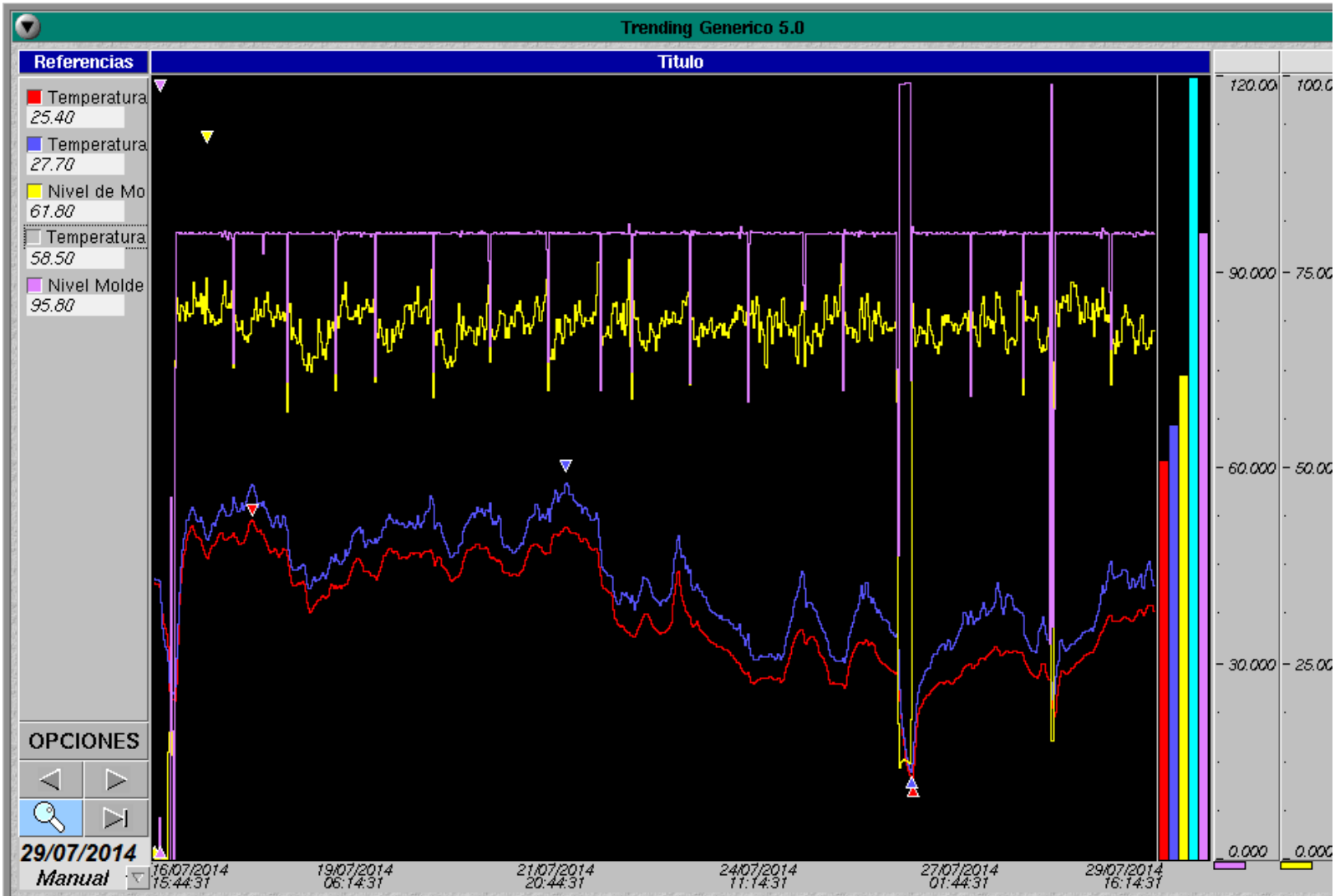

Figura 13. Trending de variables inicio de secuencia

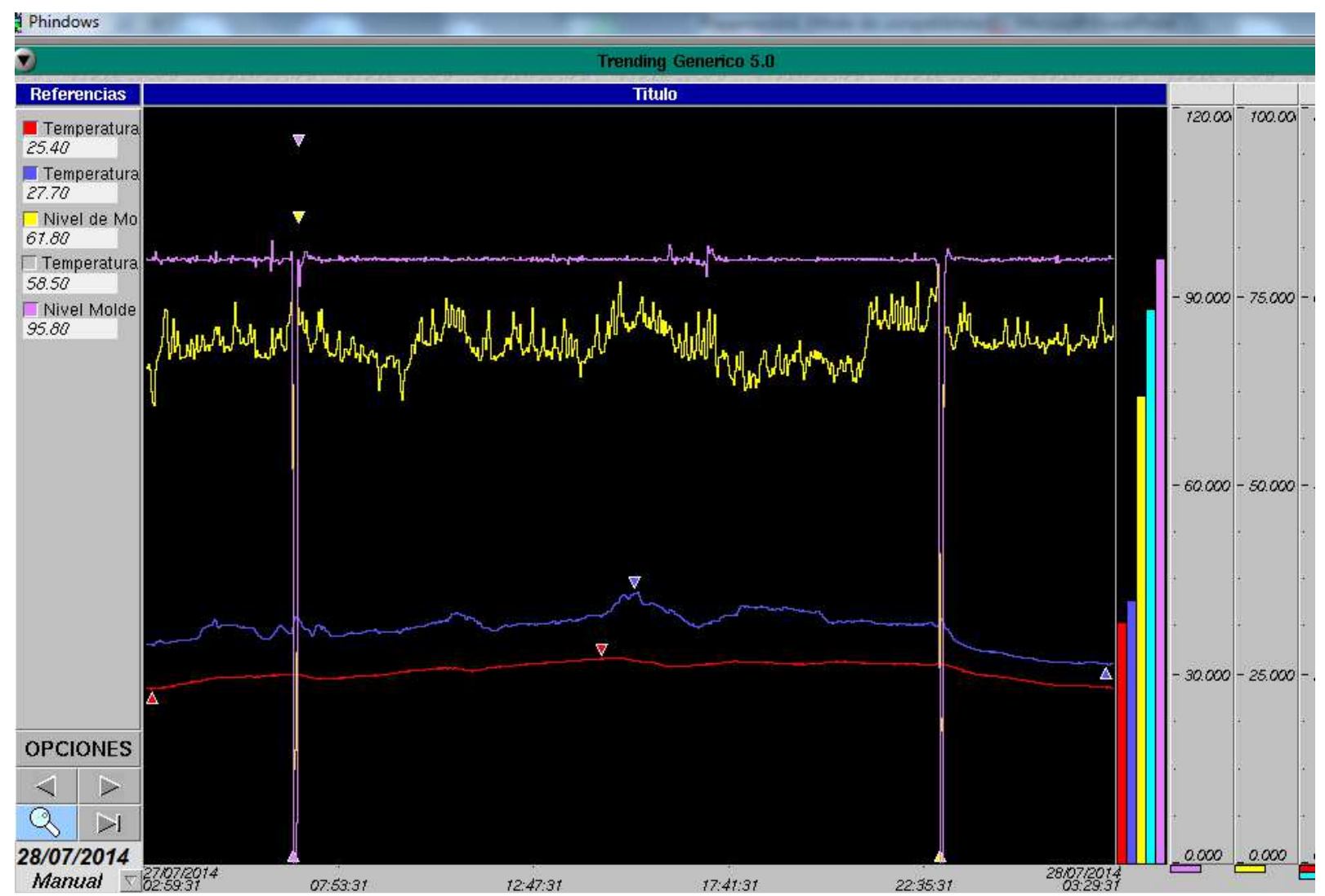

Figura 14. Trending de variables (un zoom de la anterior) 


\section{RESULTADOS Y DISCUSIÓN}

Esta etapa trajo una relativa mejora en los aspectos mencionados (calidad y continuidad operativa) pero el hecho de tener como sistema de medición de nivel un sistema radiactivo (Fuente Co60 y contador de centelleo) mantiene serias limitaciones en ambos rubros dado que este sistema no diferencia la interface escoria /polvo colador con el menisco de acero.

\section{CONCLUSIÓN}

Este desarrollo y resultados mencionados nos lleva a un desafío que a partir de un futuro cercano, el Control de Nivel de acero de las dos coladas continuas de Ternium-Siderar sean a partir de medición de nivel exclusiva de sistema electromagnético

\section{REFERENCIAS}

1 45th Steelmaking Seminar - International of the ABM, Raul Daniel López y Jaime Usart. Ternium-Siderar Slab Continuous Caster №1, 20 years of technological changes. May 26th-28th 2014, Porto Alegre - RS, Brazil.

2 19th IAS Steel Conference 2013, International Course, Arthur Vaterlaus (Switzerland) Continuous Casting Technology, November 04th 2013, Rosario, Pcia. de Santa Fe. República Argentina 